\title{
Evaluation of oxidative stress parameters in dogs with brachycephalic obstructive airway syndrome before and after surgery
}

\author{
Vladimira Erjavec ${ }^{1}$, Tomaž Vovk ${ }^{2}$, Alenka Nemec Svete ${ }^{1 凶}$ \\ ${ }^{1}$ Small Animal Clinic, Veterinary Faculty, University of Ljubljana, Ljubljana, 1000, Slovenia \\ ${ }^{2}$ The Chair of Biopharmaceutics and Pharmacokinetics, Faculty of Pharmacy, University of Ljubljana, Ljubljana, 1000, Slovenia \\ alenka.nemecsvete@vf.uni-lj.si
}

Received: September 30, $2020 \quad$ Accepted: May 7, 2021

\begin{abstract}
Introduction: Canine brachycephalic obstructive airway syndrome (BOAS) is a conformation-related respiratory disorder of dog breeds having congenitally flattened facial and skull anatomy. The aim of the study was to determine oxidative stress parameters, the lipid peroxidation product malondialdehyde, and antioxidant enzymes superoxide dismutase (SOD) and glutathione peroxidase in BOAS patients before and after surgical treatment and in healthy brachycephalic dogs. Material and Methods: Nine healthy brachycephalic dogs that had not undergone surgery and 39 BOAS patients were included in the study. The BOAS patients were classified as grade $1(5 / 34)$, grade $2(16 / 34)$, and grade $3(13 / 34)$ based on the decrease in the radius of the airway in the larynx. In BOAS patients, oxidative stress parameters were determined before and two weeks after surgery, while in control dogs, blood samples were collected only on inclusion to the study. Results: All BOAS patients showed various degrees of improvement in clinical signs after surgery. Significantly lower $(\mathrm{P}<0.05)$ SOD activity was found in grade 2 and 3 BOAS patients than in grade 1 patients. Two weeks after surgery, a significant $(\mathrm{P}<0.05)$ increase in SOD activity in grade 2 and 3 patients was observed. Conclusion: Antioxidant enzyme SOD may play an important role in BOAS and can be used as a biomarker of antioxidant status assessment in BOAS patients.
\end{abstract}

Keywords: brachycephalic obstructive airway syndrome, malondialdehyde, superoxide dismutase, glutathione peroxidase, oral surgery.

\section{Introduction}

In recent years, brachycephalic dogs have become increasingly popular, possibly due to the similarity between the head shape of these breeds and that of human infants and their being generally less fearful of strangers than dolichocephalic (long, narrowskulled) breeds (20-22). Bulldogs, pugs, and Boston terriers are examples of extremely brachycephalic breeds characterised by 'severe shortening of the muzzle, and therefore the underlying bones, and a more modest shortening and widening of the skull' as Bannasch et al. described (3). Breeding selection for extreme brachycephaly has resulted in the deformation of the upper airway leading to obstruction, as the soft tissues have not reduced proportionally with the length of the skull $(8,13,22)$.

Brachycephalic dogs are prone to a conformationrelated respiratory disorder known as brachycephalic obstructive airway syndrome (BOAS), which is a progressive disease $(8,10,19,21,22,24)$. Affected dogs show clinical signs that may include respiratory distress, such as inspiratory dyspnoea, stertor, stridor and panting, stress, exercise and heat intolerance, cyanosis and even syncopal episodes, gastrointestinal problems, snoring and disturbed sleep patterns. Brachycephalic dog breeds may have additional systemic complications. Besides, BOAS has potentially severe consequences for their welfare $(8,21,23,24,26)$.

Treatment of affected dogs can be surgical or conservative $(8,21)$. However, conservative treatment is merely palliative and surgical intervention is recommended in most clinically affected dogs (30). Current standard surgical options for the treatment of BOAS combine staphylectomy, alaplasty with or without vestibuloplasty, and in some cases, excision of everted laryngeal saccules $(8,12,30)$. Studies suggest that surgical treatment of BOAS is associated with long-term improvement in 
clinical signs regardless of age, breed and specific diagnoses $(8,11,12,25)$. Surgical resection is the preferred procedure to treat stenotic nares and elongated soft palates in brachycephalic dogs $(8,11,12,25,30)$.

Brachycephalic obstructive airway syndrome shares features of obstructive sleep apnoea syndrome (OSAS) $(13,14)$, which is the most common form of sleep-disordered breathing in people (9). Obstructive sleep apnoea syndrome is caused by the repetitive collapse of the narrow upper airway during sleep. Patients with OSAS experience repeated episodes of cessation of breathing, leading to hypoxia and reoxygenation. These events can cause the increased generation of reactive oxygen species (ROS) and reactive nitrogen species and thus oxidative stress, which may further lead to endothelial dysfunction, vascular inflammation, and atherosclerosis, playing a major role in the progression of cardiovascular disease in OSAS patients $(9,15)$. Several researchers have reported changes of levels of oxidative stress parameters indicative of increased oxidative stress in OSAS patients $(6,9,17)$.

To the authors' knowledge, oxidative stress parameters have not been investigated in brachycephalic dogs. The aim of the present study was to determine oxidative stress parameters, i.e. the concentration of the lipid peroxidation product malondialdehyde (MDA) and the activity of the two major intracellular antioxidant enzymes superoxide dismutase (SOD) and glutathione peroxidase (GPX) in BOAS patients before and after the surgical treatment of BOAS and in systemically healthy brachycephalic dogs.

\section{Material and Methods}

Animals. In total, 49 client-owned dogs of brachycephalic breeds were assessed during the study period. Thirty-nine dogs were prospectively recruited as BOAS patients, and ten systemically age-matched, healthy dogs of brachycephalic breeds that did not undergo surgery served as controls. The 10 control dogs were judged to be healthy based upon normal history, clinical examination finding no abnormalities, and the results of haematological and biochemical analyses (results not shown).

In the initial presentation, the history of the dogs was collected using a questionnaire about behaviour, health, and lifestyle. All dogs that had any signs of concurrent disease or had received any therapy, vaccination or supplementation with vitamins and other antioxidants within the previous month were excluded from the study. It transpired that five BOAS patients were excluded due to the presence of concurrent diseases. A preoperative owner questionnaire on BOASrelated signs (respiratory signs, gastrointestinal signs, exercise intolerance, and sleep disorders) modified from Roedler et al. (26) was completed for each dog. Each question response regarding a particular clinical sign was graded from 1 to 5 (' 1 ' meaning that the clinical sign was not present and ' 5 ' meaning that the clinical sign was always present). The questionnaire was reassessed two weeks after surgery.

Brachycephalic obstructive airway syndrome diagnosis was made based on clinical signs of upper airway obstruction and anatomical abnormalities, as has been described elsewhere $(8,13)$. The affected dogs included in the study did not present any other illness apart from BOAS. Disease severity was classified according to nasopharyngeal and laryngeal dimensions and obstructions at different anatomical levels such as abnormal conchal growth or turbinate protrusion, redundant and hypertrophied pharyngeal folds, enlarged and everted tonsils, macroglossia, and laryngeal oedema or collapse $(8,13)$. Patients were classified as grade 1 , grade 2 , or grade 3 based on the decrease of the airway diameter at the level of the nasopharynx and of the larynx. Grade 1 patients $(5 / 34 ; 14.7 \%)$ had no or very mild narrowing of the airway caused by mild eversion of the laryngeal saccules or mild medial displacement of the cuneiform and corniculate cartilage, no eversion of the tonsils, and no or mild nasopharyngeal obstruction. Grade 2 patients $(16 / 34 ; 47.1 \%)$ had a 50\% decrease in the radius of the airway caused by moderate nasopharyngeal obstruction, moderate eversion of the laryngeal saccules and displacement or overlapping of the cuneiform processes, moderate macroglossia, and enlarged and everted tonsils. Grade 3 patients (13/34; $38 \%$ ) had obstruction of the airway at several anatomical levels, such as at the level of the nasopharynx caused by abnormal conchal growth with a turbinate protrusion, at the level of the pharynx caused by overlong soft palate redundant and hypertrophied pharyngeal folds, enlarged and everted tonsils and severe macroglossia, and/or at the level of the larynx caused by severe overlapping of the cuneiform processes and severe eversion of laryngeal saccules. Patients with redundant and hypertrophied pharyngeal folds, a very massive base of the tongue, and limited opening of the mouth but that otherwise did not have a severe obstruction at the level of the larynx were also included in the grade 3 classification. These patients would normally be classified as grade 1 or 2 by the measure of their laryngeal collapse. Patients in all groups had an elongated soft palate, and most patients in grade 2 had eversion of the tonsils. Dogs with syndrome were scheduled for surgical treatment under general anaesthesia. The health status of the patients was evaluated based on history, a physical examination, and blood tests including haematological and biochemical analyses (results not shown).

The formal written consent of the owner was obtained before the dogs entered the study. All procedures complied with the relevant Slovenian governmental regulations (Animal Protection Act UL RS, 43/2007). The study was evaluated and approved by the Ethical Committee on Animal Research of the Veterinary Faculty, University of Ljubljana.

Anaesthesia and anatomical evaluation. Food was withheld for 14 hours prior to anaesthesia; access to 
water was allowed until premedication. Dogs were premedicated with midazolam $0.1 \mathrm{mg} / \mathrm{kg}$ b.w. IV (Midazolam Torrex; Chiesi Pharmaceuticals $\mathrm{GmbH}$, Vienna, Austria) and butorphanol $0.2 \mathrm{mg} / \mathrm{kg}$ b.w. IV (Butomidor; Richter Pharma, Wels, Austria) 2-5 minutes before induction of anaesthesia. Dogs were placed in sternal recumbency. Upper airways were examined after induction of the light plane of IV anaesthesia using propofol 3-6 mL/kg (Norofol; Norbrook Laboratories Ltd, Newry, UK). The tonsils, soft palate, larynx, and trachea were evaluated by rigid endoscopy. Assessment of the nares was after induction of anaesthesia when breathing was not laboured and stenosis of the nares became more severe; the grading was as mildly, moderately, or severely stenotic (19). For nasopharynx evaluation by a flexible endoscope, anaesthesia was deepened. After anatomical evaluation, the dogs were intubated, connected to a circle breathing system, and allowed to breathe oxygen spontaneously. Anaesthesia was maintained with sevoflurane (Sevorane; Abbott Laboratories, Sittingbourne, UK) at an end-tidal concentration of $2-3 \%$. Hartmann's solution (B Braun; Melsungen, Germany) was infused at $5 \mathrm{~mL} / \mathrm{kg} / \mathrm{h} \mathrm{IV}$. End-tidal sevoflurane concentration, end-tidal carbon dioxide tension, respiratory rate, ECG, blood pressure (non-invasively), and rectal temperature were monitored during anaesthesia.

All dogs recovered from general anaesthesia and were extubated and returned to their owners when they were fully awake. During recovery, the dogs were administered Hartmann's solution at $2 \mathrm{~mL} / \mathrm{kg} / \mathrm{h}$ IV and heptanone (Syntadone; Le Vet Behreer B.V., Utrecht, the Netherlands) at $0.3 \mathrm{mg} / \mathrm{kg}$ subcutaneously 2-4 hours after premedication. Depending on gastrointestinal tract history, dogs were administered Carprofen (Rimadyl; Orion Pharma Animal Health, Sollentuna, Sweden) at $4 \mathrm{mg} / \mathrm{kg} \mathrm{IV}$, meloxicam (Loxicom; Norbrook Laboratories Limited), or Tramadol (Tramal; STADA Arzneimittel, Bad Vilbel, Germany) at 3-5 mg/kg. All dogs were released to home care the same day, with the analgaesics listed above prescribed as clinically indicated. Antacid as $0.7 \mathrm{mg} / \mathrm{kg}$ omeprazole (Nexium; AstraZeneca AB, Gothenburg, Sweden) orally every 24 hours and prokinetic medication of $0.2 \mathrm{mg} / \mathrm{kg}$ metoclopramide (Reglan, ALKALOID-INT d.o.o., Ljubljana, Slovenia) every 8 hours for 14 days were also prescribed. Dexamethasone (Dexamethason Krka; KRKA, d.d., Novo Mesto, Slovenia) at $0.1 \mathrm{mg} / \mathrm{kg}$ was given IV during anaesthesia and $4 \mathrm{~h}$ later, if necessary. Dogs with nausea after recovery were administered $1 \mathrm{mg} / \mathrm{kg}$ maropitant citrate (Cerenia; Zoetis, Ottignies-Louvainla-Neuve, Belgium). Antibiotics were not given unless aspiration pneumonia was suspected.

Surgical procedure. Nare resection was performed using a horizontal wedge excision of the dorsomedial and caudal portion of the alae with a No. 11 scalpel blade. Two to four simple interrupted sutures using the synthetic absorbable monofilament suture material Glycomer 631(Biosyn 4/0; Covidien, Dublin, Ireland) were placed to oppose the wedge margins. Because many brachycephalic dogs have an excessively thick as well as long soft palate, folded flap palatoplasty was performed in all dogs. Each dog was placed in a sternal position. A portion of the oropharyngeal mucosa and underlying soft tissue was excised using a Surgitron Radiolase II (Ellman International, Inc., Hicksville, NY, USA). In 3 of the 32 dogs, a sacculectomy was additionally performed.

Blood sampling and processing. In BOAS dogs, venous blood samples were collected from the cephalic vein twice, 2 hours ( \pm 15 minutes) before premedication and oxygen supplementation (basal values) and two weeks after surgical treatment. Control dogs were not subjected to surgical treatment, so venous blood samples were collected once to obtain basal values. Blood samples for haematological analyses (a complete blood count and white blood cell differential count) were collected into tubes containing the anticoagulant ethylenediaminetetraacetic acid (EDTA) (BD Microtainer; Becton Dickinson, Franklin Lakes, NJ, USA). Blood samples for determining the biochemical profile (glucose, urea, creatinine, sodium, potassium, chloride, total protein, albumin, alanine aminotransferase, and alkaline phosphatase) were collected into serum separator tubes (Vacuette; Greiner Bio-One, Kremsmünster, Austria). Blood samples were centrifuged at 1,300 $\times g$ for $10 \mathrm{~min}$ at room temperature. Venous blood samples for determination of whole blood GPX and SOD activities were collected into Vacutainer tubes containing lithium heparin (Vacutainer Systems; Becton Dickinson), as recommended by the manufacturer of the reagent kits that were used for their determination. Samples of heparinised whole blood were aliquoted and immediately frozen at $-80^{\circ} \mathrm{C}$ until analysis. Blood samples for determining plasma concentrations of MDA were collected into Vacuette tubes containing EDTA. Samples were centrifuged immediately after collection at $1,500 \times g$ for $15 \mathrm{~min}$ at $4^{\circ} \mathrm{C}$. Plasma was harvested, aliquoted, and immediately frozen at $-80^{\circ} \mathrm{C}$ until analysis.

Haematological and biochemical analyses. Haematological analyses were performed within $1 \mathrm{~h}$ of collection of the blood samples using an automated laser-based haematology analyser (ADVIA 120; Siemens, Munich, Germany) and multispecies software. The biochemical profiles were determined on the day of sample collection. Except for electrolytes, biochemical profiles were determined with an automated biochemical analyser (RX Daytona; Randox, Crumlin, UK). The concentrations of electrolytes were determined with an electrolyte analyser (ILyte, Instrumentation Laboratory, Lexington, MA, USA).

Determination of GPX and SOD activities. Activities of whole blood GPX and SOD were determined spectrophotometrically with the RX Daytona biochemical analyser using the commercially available Ransel and Ransod kits (both Randox), respectively. These were expressed as units per gram of haemoglobin (U/g Hgb).

Determination of MDA concentration. Total MDA plasma concentration was determined using the 
derivatisation method with 2, 4-dinitrophenylhydrazine (7). Derivatised plasma samples were injected into an Agilent 1200 series high-performance liquid chromatography system (Agilent, Waldbronn, Germany) and separated on an Agilent Eclipse XBD-C18 column $(5 \mu \mathrm{m}, 150 \mathrm{~mm}$ $\times 4.6 \mathrm{~mm}$ ) using gradient elution with acetonitrile, water and acetic acid (38:62:0.2\%, v/v/v) as the initial mobile phase. The MDA derivative was detected with the ultraviolet detector at $310 \mathrm{~nm}$.

Statistical analysis. Data were analysed with commercial software (IBM SPSS 22.0, Armonk, NY, USA). Descriptive statistics were used to describe the basic features of the data. The Shapiro-Wilk test was performed to test whether the data were normally distributed. A one-way ANOVA with Tukey honestly significant difference post-hoc test in the case of normal distribution of the data or Kruskal-Wallis test followed by multiple comparisons in the case of non-normal distribution of the data were used to test for statistically significant differences in the measured parameters (including age and weight) among the groups of dogs (three groups of BOAS patients and the control group) before and after surgery. In each group of BOAS patients, a paired $t$-test in the case of normal distribution of the data or the Wilcoxon rank-sum test in the case of non-normal distribution of the data was performed to test for statistically significant differences in the measured parameters and the sum of individual sets of clinical signs (respiratory, gastrointestinal, sleep disorders, and exercise intolerance) before and after surgical treatment. Pearson's correlation analysis was performed to determine the correlation between laryngeal collapse and individual sets of clinical signs. The data that were normally distributed are reported as means $\pm \mathrm{SD}$; the data that were not normally distributed are reported as medians and interquartile range (IQR -25 th to 75 th percentile). A value of $\mathrm{P}<0.05$ was considered significant.

\section{Results}

In the present study, 49 dogs were evaluated; however, 5 BOAS patients and 1 control dog were excluded from the study due to the presence of concurrent diseases. Thus, 34 dogs with confirmed BOAS and 9 control dogs were enrolled in the study. Brachycephalic obstructive airway syndrome patients were of the following breeds: French bulldog $(n=14)$, pug $(n=9)$, Boston terrier $(n=5)$, English bulldog $(n=3)$, Yorkshire terrier $(n=2)$ and Japanese chin $(n=1)$. The following breeds were included in the control group: French bulldog $(n=6)$, Boston terrier $(n=2)$ and Cavalier King Charles spaniel $(n=1)$. The baseline characteristics of BOAS-affected dogs are summarised in Table 1. There were no significant differences between the groups of dogs in age and weight.

The owner questionnaire revealed that brachycephalic dogs experienced severe respiratory and gastrointestinal symptoms, as well as exercise intolerance and sleep disorders. Two weeks after surgical treatment, all BOAS patients showed various degrees of improvement in all clinical signs (Table 2). The owners had to choose only the single most restrictive problem for their dog out of five possibilities. Before surgery, exercise intolerance was the predominant problem in $1 \mathrm{dog}(1 / 34 ; 3 \%)$, breathing problems in 22 dogs $(22 / 34 ; 65 \%)$, heat intolerance in 5 dogs $(5 / 34$; $15 \%)$, sleep disorders in $2 \mathrm{dogs}(2 / 34 ; 6 \%)$ and food prehension in 3 dogs $(3 / 34 ; 9 \%)$. It needs to be emphasised that all dogs had multiple problems.

Upper airway inspection showed some degree of anatomical abnormality in all BOAS patients. The most common components of BOAS were an elongated soft palate $(34 / 34 ; 100 \%)$, stenotic nares $(32 / 34 ; 94 \%)$, everted laryngeal saccules $(32 / 34 ; 94 \%)$ and everted aberrant conchae $(15 / 34 ; 44 \%)$. The degree of laryngeal collapse was scored as previously described (16); only $1 \mathrm{dog}(3 \%)$ had no laryngeal collapse, 18/34 (53\%) dogs had grade 1, 14/34 (42\%) dogs had grade 2 and only $1 \operatorname{dog}(3 \%)$ had grade 3 laryngeal collapse. No significant correlations between the severity of laryngeal collapse and overall respiratory and gastrointestinal clinical signs, sleep disorders, or exercise intolerance before and after surgery were found. There were only mild complications during surgical treatment. In 5 out of 34 dogs, the flap was perforated and sutured with synthetic absorbable monofilament suture material. Some dogs experienced breathing difficulties after extubation, and some dogs returned to the clinic due to breathing difficulties within three days of the surgery.

Parameters of oxidative stress in BOAS patients before and after surgery and in the control group are shown in Table 3.

The highest SOD activity was found in grade 1 BOAS-affected dogs before and after surgery. Statistical analysis showed significantly higher SOD activity in grade 2 BOAS-affected dogs $(\mathrm{P}=0.039)$ and grade 3 dogs $(\mathrm{P}=0.012)$ after surgery than before surgery. On the other hand, we observed no significant differences between GPX activity or MDA concentration before surgery and this activity and concentration after surgery in any of the grades of BOAS patients. We likewise found no significant differences in GPX activity or MDA concentrations before and after surgery between the three grades of BOAS patients. However, the comparison of SOD activities between different grades of BOAS patients before surgery demonstrated significantly higher SOD activity in the grade 1 compared with the grade $2(\mathrm{P}=0.043)$ and grade 3 $(\mathrm{P}=0.038)$ groups. After surgery, no significant difference in SOD activity between the grades of BOAS patients was found. Furthermore, no significant differences in SOD and GPX activity or MDA concentrations before and after surgery between the four groups of dogs (the control and the three groups of BOAS patients) were observed. 
Table 1. Baseline characteristics in groups of brachycephalic obstructive airway syndrome patients and control group

\begin{tabular}{lllll}
\hline & Control & Grade 1 & Grade 2 & Grade 3 \\
\hline Number & 9 & 5 & 16 & 13 \\
Sex (female/male) & $4 / 5$ & $3 / 2$ & $5 / 11$ & $6 / 7$ \\
Age (years) Median; IQR & $3.25 ; 1.84-3.96$ & $3.75 ; 1.08-6.96$ & $2.88 ; 1.14-7.11$ & $1.75 ; 1.46-8.88$ \\
Weight (kg) Median; IQR & $9.50 ; 8.45-11.25$ & $9.56 ; 5.75-11.54$ & $10.20 ; 7.78-12.53$ & $9.60 ; 8.37-10.70$ \\
BCS Median; IQR & $3.0 ; 3.0-3.5$ & $3.0 ; 2.5-3.5$ & $3.0 ; 3.0-4.0$ & $3.0 ; 3.0-3.0$ \\
\hline
\end{tabular}

BCS - body condition score; IQR - interquartile range (25th to 75 th percentile)

Table 2. Clinical signs (mean) in groups of brachycephalic obstructive airway syndrome patients before and after surgery

\begin{tabular}{|c|c|c|c|c|}
\hline & $\begin{array}{c}\text { Respiratory } \\
\text { signs } \\
\end{array}$ & $\begin{array}{c}\text { Gastrointestinal } \\
\text { signs }\end{array}$ & $\begin{array}{c}\text { Exercise } \\
\text { intolerance }\end{array}$ & $\begin{array}{c}\text { Sleep } \\
\text { disorders }\end{array}$ \\
\hline \multicolumn{5}{|l|}{ Grade 1} \\
\hline Before surgery & 3.1 & 1.8 & 2.1 & 3.7 \\
\hline After surgery & $1.5^{\mathrm{a}}$ & 1.2 & 1.0 & $2.0^{\mathrm{a}}$ \\
\hline \multicolumn{5}{|l|}{ Grade 2} \\
\hline Before surgery & 3.2 & 2.3 & 1.8 & 4.1 \\
\hline After surgery & $1.8^{\mathrm{a}}$ & $1.6^{\mathrm{a}}$ & 1.5 & $2.4^{\mathrm{a}}$ \\
\hline \multicolumn{5}{|l|}{ Grade 3} \\
\hline Before surgery & 3.1 & 2.1 & 1.8 & 3.8 \\
\hline After surgery & $1.9^{\mathrm{a}}$ & $1.4^{\mathrm{a}}$ & $1.2^{\mathrm{a}}$ & $2.7^{\mathrm{a}}$ \\
\hline
\end{tabular}

${ }^{\text {a }}$ Significant $(\mathrm{P}<0.05)$ difference in comparison to values before surgery

1.0 means that the clinical sign was not present and 5.0 means that the clinical

sign was always present

Table 3. Oxidative stress parameters (mean \pm standard deviation) in groups of brachycephalic obstructive airway syndrome patients before and after surgery and in the control group

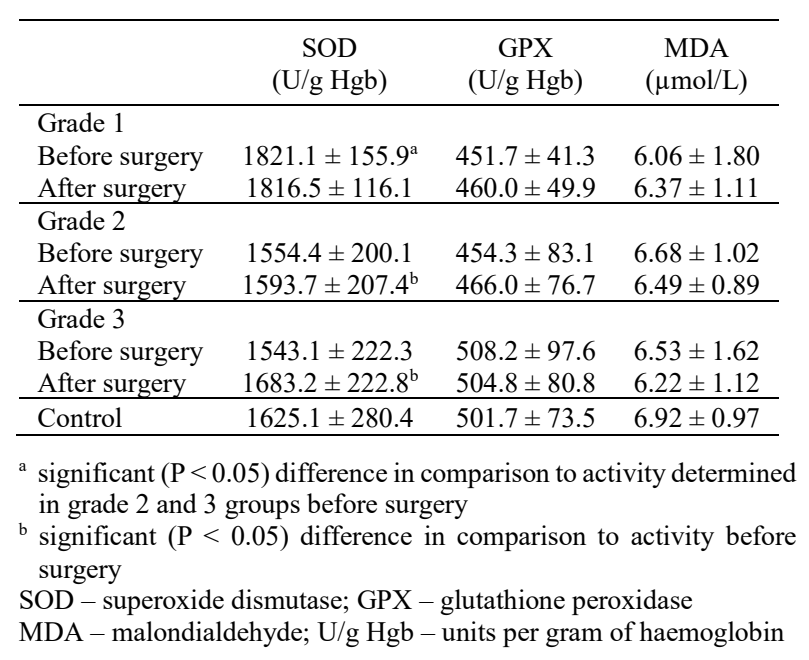

\section{Discussion}

To the authors' knowledge, the present research is the first clinical study that has investigated oxidative stress parameters in BOAS patients before and after surgical treatment. Thus, it was not possible to compare these results to those of similar studies conducted in BOAS patients. The oxidative stress parameter values obtained in the present study were compared with those of studies conducted in human OSAS patients, as most of the systemic consequences are similar. Although BOAS is not identical to OSAS, there is much common to both. Like OSAS patients, brachycephalic dogs snore and are frequently aroused from sleep due to hypoxaemia and apnoea (14). In English bulldogs, long periods of haemoglobin oxygen saturation levels less than $90 \%$ have been observed during sleep, with episodes of central and obstructive apnoea (14). Additionally, during the awake state brachycephalic dogs have episodes of severe hypoxia during exercise, stress, or heat exposure $(13,14)$. Obstructive sleep apnoea syndrome is characterised by intermittent hypoxia that leads to blood hypoxaemia, hypercapnia, sleep fragmentation, and consequently to oxidative stress and concomitant inflammation, which adversely affect the associated cardio- and cerebrovascular disease in OSAS $(9,15)$. It has been suggested that because of profound hypoxic episodes followed by rapid blood oxygenations, intermittent hypoxia could be considered analogous to repeated ischaemia and reperfusion events that result in injury due to ROS flux during the reperfusion period. Ischaemia-reperfusion injury is a well-established pathway leading to increased ROS production and thus oxidative stress (15).

It has been reported that the release of superoxide anions from circulating neutrophils and monocytes of OSAS patients is markedly potentiated in comparison with control subjects (27). Furthermore, impaired antioxidant capacity and decreased activity of antioxidant enzymes, catalase, and SOD and GPX, as well as 
increased levels of markers of lipid peroxidation, protein carbonylation, and DNA oxidation have been found in OSAS patients $(9,15,17)$. On the other hand, some studies have not demonstrated changes in parameters that could be indicative of oxidative stress in OSAS patients $(1,28)$. The results of the present study demonstrated significantly lower SOD activity in grade 2 and grade 3 patients than in grade 1 patients before surgery. In the two higher-graded groups, SOD activity increased significantly after surgical treatment, which cancelled the significance in the difference in SOD activity between the BOAS-affected dogs at that stage. On the other hand, we found no significant difference in SOD activity when comparing the three groups of BOAS patients and the control group before surgery. The activities of SOD in all four groups of dogs were in general agreement with previously reported results obtained in healthy dogs (29); however, the mean values of SOD activity in grades 2 and 3 BOAS patients before surgery were much lower than the mean or median values which were their precedents (29). As already stated, BOAS is not identical to OSAS, but there are some similarities (14). Intermittent hypoxia, which is one of the major characteristics of OSAS, results in recurrent hypoxia/reoxygenation events leading to oxidative stress (15). Thus, low SOD activity in grade 2 and grade 3 patients could be a result of increased oxidative stress due to recurrent hypoxia/reoxygenation events in these patients. Similarly to our results, low SOD activities were reported in OSAS patients and Sprague Dawley rats, the latter serving as an animal model of intermittent hypoxia $(9,15,18)$. Moreover, Cofta et al. (6) reported significantly lower SOD activity and significantly higher concentration of thiobarbituric acid reactive substances (TBARS) in OSAS patients when compared to the control group, this TBARS being noted marker of lipid peroxidation. The authors stated that oxidative stress increases with the severity of OSAS and that blood SOD activity and MDA concentration can be used to investigate oxidative stress in OSAS patients. Another reason for the significantly lower SOD activity in grade 2 and 3 patients in comparison with grade 1 might be a reduced demand for SOD as a result of reduced oxygen consumption in patients with moderate and severe BOAS. The lower consumption rate can be supposed because it would be commensurate with these patients having more pronounced respiratory signs than grade 1 patients. Overall, our results suggest that SOD may play an important role in BOAS patients and can be used as a biomarker of antioxidant status assessment in these patients.

The results of oxidative stress in OSAS patients $(2,6,9,17)$, and the shared progressive nature of OSAS and BOAS, would seem to validate our expectation that patients with different grades of BOAS would differ significantly not only in the activity of SOD but also in the activity of GPX and plasma MDA concentration. However, the GPX activity did not differ significantly between groups of BOAS patients before surgical treatment. The surgical treatment did not affect the activity of this antioxidant enzyme in any of the groups of BOAS-affected dogs. We also did not find a significant difference in GPX activity when comparing the four groups of brachycephalic dogs. These activity levels were in accordance with those in our previous results obtained in healthy dogs (29). The results might indicate that in contrast to SOD, GPX does not play a role in BOAS. Additionally, in OSAS patients, GPX activity measurement has shown contradicting results; some studies have found significantly decreased serum or erythrocyte activity of GPX in comparison with healthy subjects $(2,5)$, while others have not found significant differences (4).

Similarly to GPX, the present study did not demonstrate a significant difference in MDA concentration between the four groups of brachycephalic dogs or the three grades of BOAS patients before surgical treatment. Furthermore, the concentration of MDA was unaffected by surgical treatment in all three grades of BOAS patients. These results might indicate that no oxidative damage to lipids occurs as a consequence of increased oxidative stress in any of the disease severity groups of BOAS patients. Our results for the lipid peroxidation marker MDA, are in general agreement with those of some studies conducted on OSAS patients, where the concentrations of lipid peroxidation markers did not differ from those of healthy subjects or between pre- and post-treatment conditions $(1,28)$.

The control group consisted of clinically healthy brachycephalic dogs, which might be the reason for the lack of significant differences in oxidative stress parameters between individual groups of BOAS patients and the control group before surgery. It has been established that almost $60 \%$ of the owners of affected animals claimed that their dogs did not have a breathing problem (23); therefore, it is possible that subclinical obstruction may have existed in many if not all animals in the control group.

In the present study, surgical treatment resulted in significant improvement in clinical signs in all groups of BOAS patients, which is in accordance with other studies $(8,12,25,30)$. Dogs were divided into three groups: the grade 1 group of BOAS patients had no or very mild narrowing of the upper airway passage after removal of the elongated soft palate, the grade 2 group of patients had narrowing of the airway of up to $50 \%$ after removal of the elongated soft palate and the grade 3 group of patients had almost complete obstruction of the upper airway passage which persisted after surgery due to abnormal conchal growth with a turbinate protrusion in the nasopharynx, redundant and hypertrophied pharyngeal folds, enlarged and everted tonsils, enlarged tongue, narrow oropharynx, everted laryngeal saccules and/or collapsed larynx. Although obstruction of the airway remained at different anatomical levels, opening the nostrils and removing redundant soft palate tissue resulted in a marked improvement in clinical signs in grade 3 patients. Even 
in these dogs, a small increase in the diameter of the airway is significant, given that doubling the diameter of an airway increases the flow rate of air 16-fold. In the present study, we found no correlations between the severity of laryngeal collapse and overall respiratory and gastrointestinal clinical signs, sleep disorders or exercise intolerance before and after surgery, which is consistent with the findings of Haimel and Dupré (12).

The present study has some limitations. The first is that the low number of grade 1 patients might have affected our results. The second is the impossibility of including in the control group brachycephalic dogs assessed as healthy based on the evaluation of the anatomical abnormalities of the airway under general anaesthesia. The third limitation is the lack of a group of non-brachycephalic dog breeds, which could have been helpful in the interpretation of our results.

In conclusion, the results of the present study suggest that the antioxidant enzyme SOD may play an important role in BOAS and can be used as a biomarker of antioxidant status assessment in BOAS patients. Surgical treatment to correct narrow nostrils and obstructive soft palate conformation was indicated in all groups of BOAS patients because after it was performed the clinical signs were significantly reduced in all ages and all groups of dogs. The results of the present study warrant further studies in larger groups of BOAS patients, the inclusion of a group of nonbrachycephalic dog breeds, and the measurement of other markers of oxidative stress.

Conflict of Interests Statement: The authors declare that there is no conflict of interests regarding the publication of this article.

Financial Disclosure Statement: This work was financially supported by the Slovenian Research Agency (Program group P4-0053 and P1-0189).

Animal Rights Statement: The authors declare that the experiments on animals were conducted in accordance with local Ethical Committee laws and regulations as regards care and use of laboratory animals.

Acknowledgments: The authors thank Maruša Škrbec and Lucija Podvršnik for their help regarding blood sample processing and laboratory technician Aleksander Jenko for routine haematology and biochemistry analyses.

\section{References}

1. Alzoghaibi M.A., Bahammam A.S.O.: Lipid peroxides, superoxide dismutase and circulating IL-8 and GCP-2 in patients with severe obstructive sleep apnea: a pilot study. Sleep Breath 2005, 9, 119-126, doi: 10.1007/s11325-005-0022-1.

2. Asker S., Asker M., Sarikaya E., Sunnetcioglu A., Aslan M., Demir H.: Oxidative stress parameters and their correlation with clinical, metabolic and polysomnographic parameters in severe obstructive sleep apnea syndrome. Int J Clin Exp Med 2015, 8, 114491-11455.

3. Bannasch D., Young A., Myers J., Truvé K., Dickinson P., Gregg J., Davis R., Bongcam-Rudloff E., Webster M.T., Lindblad-Toh K., Pedersen N.: Localization of canine brachycephaly using an across breed mapping approach. PLoS One 2010, 5, e9632, doi: 10.1371/journal.pone.0009632.

4. Barceló A., Barbé F., de la Peña M., Vila M., Pérez G., Piérola J., Durán J., Agustí A.G.N.: Antioxidant status in patients with sleep apnoea and impact of continuous positive airway pressure treatment. Eur Respir J 2006, 27, 756-760, doi: 10.1183/09031936.06.00067605.

5. Chen P.C., Guo C.H., Tseng C.J., Wang K.C., Liu P.J.: Blood trace minerals concentrations and oxidative stress in patients with obstructive sleep apnea. J Nutr Health Aging 2013, 17, 639-644, doi: 10.1007/s12603-013-0023-x.

6. Cofta S., Wysocka E., Piorunek T., Rzymkowska M., BaturaGabryel H., Torlinski L.: Oxidative stress markers in the blood of persons with different stages of obstructive sleep apnea syndrome. J Physiol Pharmacol 2008, 59 Suppl 6, 183-190.

7. Czauderna M., Kowalczyk J., Marounek M.: The simple and sensitive measurement of malondialdehyde in selected specimens of biological origin and some feed by reversed phase high performance liquid chromatography. J Chromatogr B 2011, 879, 2251-2258, doi: 10.1016/j.jchromb.2011.06.008.

8. Dupré G., Heidenreich D.: Brachycephalic Syndrome. Vet Clin North Am Small Anim Pract 2016, 46, 691-707, doi: 10.1016/j.cvsm.2016.02.002.

9. Eisele H.-J., Markart P., Schulz R.: Obstructive Sleep Apnea, Oxidative Stress, and Cardiovascular Disease: Evidence from Human Studies. Oxid Med Cell Longev 2015, 2015, 608438, doi: $10.1155 / 2015 / 608438$.

10. Fasanella F.J., Shivley J.M., Wardlaw J.L., Givaruangsawat S.: Brachycephalic airway obstructive syndrome in dogs: 90 cases (1991-2008). J Am Vet Med Assoc 2010, 237, 1048-1051, doi: 10.2460/javma.237.9.1048

11. Findji L., Dupré G.: Folded flap palatoplasty for treatment of elongated soft palates in 55 dogs. Eur J Companion Anim Pract 2009, 19, 125-132.

12. Haimel G., Dupré G.: Brachycephalic airway syndrome: a comparative study between pugs and French bulldogs. J Small Anim Pract 2015, 56, 714-719, doi: 10.1111/jsap.12408.

13. Hendricks J.C.: Brachycephalic airway syndrome. Vet Clin North Am Small Anim Pract 1992, 22, 1145-1153, doi.org/10.1016/S0195-5616(92)50306-0.

14. Hendricks J.C., Kline L.R., Kovalski R.J., O’Brien J.A., Morrison A.R., Pack A.I.: The English bulldog: a natural model of sleepdisordered breathing. J Appl Physiol (1985) 1987, 63, 1344-1350, doi: 10.1152/jappl.1987.63.4.1344.

15. Lavie L.: Oxidative stress in obstructive sleep apnea and intermittent hypoxia--revisited--the bad ugly and good: implications to the heart and brain. Sleep Med Rev 2015, 20, 27-45, doi: 10.1016/j.smrv.2014.07.003.

16. Leonard H.C.: Collapse of the larynx and adjacent structures in the dog. J Am Vet Med Assoc 1960, 137, 360-363.

17. Lira A.B., de Sousa Rodrigues C.F.: Evaluation of oxidative stress markers in obstructive sleep apnea syndrome and additional antioxidant therapy: a review article. Sleep Breath 2016, 20, 1155-1160, doi: 10.1007/s11325-016-1367-3.

18. Liu H. -G., Liu K., Zhou Y.-N., Xu Y. -J.: Apocynin attenuate spatial learning deficits and oxidative responses to intermittent hypoxia. Sleep Med 2010, 11, 205-212, doi: 10.1016/j.sleep. 2009.05.015.

19. Liu N.-C., Troconis E.L., Kalmar L., Price D.J., Wright H.E., Adams V.J., Sargan D.R., Ladlow J.F.: Conformational risk factors of brachycephalic obstructive airway syndrome (BOAS) in pugs, French bulldogs, and bulldogs. PLoS One 2017, 12, e0181928, doi: 10.1371/journal.pone.0181928.

20. McGreevy P.D., Georgevsky D., Carrasco J., Valenzuela M., Duffy D.L., Serpell J.A.: Dog behavior co-varies with height, 
bodyweight and skull shape. PLoS One 2013, 8, e80529, doi: 10.1371/journal.pone.0080529.

21. Meola S.D.: Brachycephalic airway syndrome. Top Companion Anim Med 2013, 28, 91-96, doi: 10.1053/j.tcam.2013.06.004.

22. O'Neill D.G., Jackson C., Guy J.H., Church D.B., McGreevy P.D., Thomson P.C., Brodbelt D.C.: Epidemiological associations between brachycephaly and upper respiratory tract disorders in dogs attending veterinary practices in England. Canine Genet Epidemiol 2015, 2, 10, doi: 10.1186/s40575-015-0023-8.

23. Packer R.M.A., Hendricks A., Burn C.C.: Do dog owners perceive the clinical signs related to conformational inherited disorders as 'normal' for the breed? A potential constraint to improving canine welfare. Animal Welfare 2012, 21, 81-93, doi: 10.7120/096272812X13345905673809.

24. Packer R.M.A., Hendricks A., Tivers M.S., Burn C.C.: Impact of Facial Conformation on Canine Health: Brachycephalic Obstructive Airway Syndrome. PLoS One 2015, 10, e0137496, doi: 10.1371/journal.pone.0137496.

25. Riecks T.W., Birchard S.J., Stephens J.A.: Surgical correction of brachycephalic syndrome in dogs: 62 cases (1991-2004). J Am Vet Med Assoc 2007, 230, 1324-1328, doi: 10.2460/javma.230.9.1324.
26. Roedler F.S., Pohl S., Oechtering G.U.: How does severe brachycephaly affect dog's lives? Results of a structured preoperative owner questionnaire. Vet J 2013, 198, 606-610, doi: 10.1016/j.tvj1.2013.09.009.

27. Schulz R., Mahmoudi S., Hattar K., Sibelius U., Olschewski H., Mayer K., Seeger W., Grimminger F.: Enhanced release of superoxide from polymorphonuclear neutrophils in obstructive sleep apnea. Impact of continuous positive airway pressure therapy. Am J Respir Crit Care Med 2000, 162, 566-570, doi: 10.1164/ajrccm.162.2.9908091.

28. Svatikova A., Wolk R., Lerman L.O., Juncos L.A., Greene E.L., McConnell J.P., Somers V.K.: Oxidative stress in obstructive sleep apnoea. Eur Heart J 2005, 26, 2435-2439, doi: 10.1093/eurheartj/ehi440.

29. Tomsic K., Seliškar A., Lukanc B., Nemec Svete A.: Plasma Total Antioxidant Capacity and Activities of Blood Glutathione Peroxidase and Superoxide Dismutase Determined in Healthy Dogs by Using Commercially Available Kits. Acta VeterinariaBeograd 2016, 66, 534-548, doi: 10.1515/acve-2016-0046.

30. Torrez C.V., Hunt G.B.: Results of surgical correction of abnormalities associated with brachycephalic airway obstruction syndrome in dogs in Australia. J Small Anim Pract 2006, 47, 150-154, doi: 10.1111/j.1748-5827.2006.00059.x. 